\title{
Kinematics, Balance Equations, and Principles of Stokes Flow
}

The focus of this book is the dynamics of fluids at small scales and of small objects (e.g., particles, cells, macromolecules) suspended in fluids. As we will see, such suspensions or solutions can have nontrivial dynamical and rheological behavior: i.e., in this regard they are complex fluids. Small is relative, of course, and we exclusively consider systems that are not so small that atomistic details of the fluid or objects are important; in particular, we treat the fluid as a continuum to which we can assign properties at every spatial position $\boldsymbol{x}$. For liquids, the continuum approximation is broadly valid for scales of about 1 nanometer (nm) and larger (a water molecule has a size of about $0.2 \mathrm{~nm}$ ). One way that we know this is through molecular simulations (Schmidt \& Skinner 2003, 2004), which show agreement with, for example, the continuum prediction for the drag force on a moving sphere even when the sphere is only several solvent atoms across. In the first several chapters of this book, we will only concern ourselves with the behavior of fluids, and particles within fluids, in the absence of thermal fluctuations. This behavior is governed by the classical equations of continuum mechanics, which are the starting point of the chapter. After reviewing these here, the governing equations for Newtonian fluids are introduced. Our ultimate focus is the Stokes equation, which governs fluid motions when the inertia of the fluid is negligible compared to viscous stresses.

\subsection{Kinematics of Continua}

\subsubsection{Velocity Fields and the Velocity Gradient}

Under the continuum approximation, a material can take on a velocity $\boldsymbol{v}$ (which may vary with time $t$ ) at every position $\boldsymbol{x}$ : i.e., we have a velocity field $\boldsymbol{v}(\boldsymbol{x}, t)$. We further

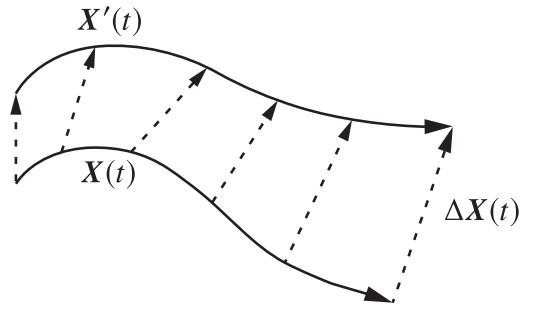

Figure 1.1 Motions of material points $X(t)$ and $\boldsymbol{X}^{\prime}(t)$, with the material line $\Delta \boldsymbol{X}(t)=\boldsymbol{X}^{\prime}(t)-\boldsymbol{X}(t)$ (dashed) shown at several time instants. 
assume that this field is differentiable except possibly at interfaces between different materials or phases. Now, consider two neighboring material points $\boldsymbol{X}$ and $\boldsymbol{X}^{\prime}$, which by definition move with the instantaneous velocity of the material at their respective positions, as illustrated in Figure 1.1. Thus

$$
\frac{d \boldsymbol{X}}{d t}=\boldsymbol{v}(\boldsymbol{X}, t), \quad \frac{d \boldsymbol{X}^{\prime}}{d t}=\boldsymbol{v}\left(\boldsymbol{X}^{\prime}, t\right) .
$$

To understand how the material deforms as it flows with velocity $\boldsymbol{v}(\boldsymbol{x}, t)$, we consider the time evolution of a "material line" $\Delta \boldsymbol{X}=\boldsymbol{X}^{\prime}-\boldsymbol{X}$ connecting points $\boldsymbol{X}$ and $\boldsymbol{X}^{\prime}$. We can then write

$$
\frac{d \Delta X}{d t}=\boldsymbol{v}\left(X^{\prime}, t\right)-\boldsymbol{v}(X, t)
$$

Now we take the distance between $\boldsymbol{X}$ and $\boldsymbol{X}^{\prime}$ to be small, so that

$$
\boldsymbol{v}\left(\boldsymbol{X}^{\prime}, t\right)=\boldsymbol{v}(\boldsymbol{X}, t)+\Delta \boldsymbol{X} \cdot \boldsymbol{\nabla} \boldsymbol{v}(\boldsymbol{X}, t)+O\left(|\Delta \boldsymbol{X}|^{2}\right),
$$

where $\boldsymbol{\nabla} \boldsymbol{v}(\boldsymbol{X}, t)$ is the velocity gradient evaluated at the material point $\boldsymbol{X}$. Combining these two equations yields that

$$
\frac{d \Delta X}{d t}=\Delta \boldsymbol{X} \cdot \nabla \boldsymbol{v}
$$

or equivalently

$$
\frac{d \Delta \boldsymbol{X}}{d t}=\mathbf{L} \cdot \Delta \boldsymbol{X}
$$

where $\mathbf{L}=\boldsymbol{\nabla} \boldsymbol{v}^{\mathrm{T}}$. Therefore, all information about the deformation of an infinitesimal line connecting two neighboring points in the fluid, is contained in the velocity gradient tensor. ${ }^{1}$ Note that we use the convention ${ }^{2}$ that in Cartesian coordinates

$$
(\boldsymbol{\nabla v})_{i j}=\frac{\partial}{\partial x_{i}} v_{j}
$$

Flows in which the velocity gradient is independent of position are called linear flows, because the velocity is a linear function of position:

$$
v(x)-v_{0}=\mathbf{L} \cdot\left(x-x_{0}\right),
$$

where $\boldsymbol{v}_{0}$ is a constant uniform velocity and $\boldsymbol{x}_{0}$ is a constant position. By appropriate choice of reference frame, we can always take $\boldsymbol{v}_{0}$ and $\boldsymbol{x}_{0}$ to be zero. If in addition $\boldsymbol{\nabla} \boldsymbol{v}$ is independent of position and time, then (1.1) is a simple linear constant coefficient equation and the evolution of $\Delta \boldsymbol{X}$ is completely determined by the eigenvalues and eigenvectors of $\boldsymbol{\nabla} \boldsymbol{v}$. We will exclusively consider incompressible flows, which satisfy

1 See Section A.1 for a brief summary of vector and tensor notation as used in this book.

2 This convention is common in the fluid mechanics literature but is not universal. In much of the continuum mechanics literature, e.g. Malvern (1969), Gonzalez \& Stuart (2008), $(\boldsymbol{\nabla v})_{i j}$ is defined as $\frac{\partial v_{i}}{\partial x_{j}}$. This is the transpose of what we use. 
$\boldsymbol{\nabla} \cdot \boldsymbol{v}=0$ due to mass conservation (see Section 1.2.1), so the eigenvalues $\lambda_{i}$ of $\boldsymbol{\nabla} \boldsymbol{v}$ must sum to zero. Equivalently,

$$
\operatorname{tr} \boldsymbol{\nabla} \boldsymbol{v}=\boldsymbol{\nabla} \cdot \boldsymbol{v}=\sum_{i=1}^{3} \lambda_{i}=0 .
$$

We will often make use of the decomposition of the velocity gradient tensor into the symmetric strain rate or deformation rate tensor $\mathbf{E}$ and antisymmetric vorticity tensor $^{3} \mathbf{W}$ :

$$
\boldsymbol{\nabla v}=\mathbf{E}+\mathbf{W}
$$

where

$$
\mathbf{E}=\frac{1}{2}\left(\boldsymbol{\nabla} v+\nabla v^{T}\right)
$$

and

$$
\mathbf{W}=\frac{1}{2}\left(\boldsymbol{\nabla} v-\nabla v^{T}\right)
$$

The vorticity vector $w$ is given by

$$
\boldsymbol{w}=\boldsymbol{\nabla} \times \boldsymbol{v}
$$

and is related to the local angular velocity of the fluid, $\omega$, by the simple expression

$$
\omega=\frac{1}{2} w
$$

These quantities are related to the vorticity tensor as follows:

$$
\mathbf{W}=\boldsymbol{\epsilon} \cdot \omega=\frac{1}{2} \epsilon \cdot w
$$

where $\boldsymbol{\epsilon}$ is the Levi-Civita symbol, whose properties are summarized in Appendix A.1.2.

If $\mathbf{E}=\mathbf{0}$ at some point in the fluid, then an infinitesimal volume of material at that point, a fluid element, is undergoing rigid rotation: there is no stretching of material lines within that volume. On the other hand, if $\mathbf{W}=\mathbf{0}$, then the fluid element is undergoing stretching without any rotation. Now $\boldsymbol{\nabla} \boldsymbol{v}$ is symmetric, so its eigenvectors are orthogonal, forming a coordinate system in which $\boldsymbol{\nabla} \boldsymbol{v}$ can be written

$$
\boldsymbol{\nabla} \boldsymbol{v}=\left[\begin{array}{ccc}
\lambda_{1} & 0 & 0 \\
0 & \lambda_{2} & 0 \\
0 & 0 & \lambda_{3}
\end{array}\right] .
$$

Defining the extension rate as $\dot{\epsilon}(>0)$, important special cases include the following:

- Uniaxial extension: $\lambda_{1}=\dot{\epsilon}, \lambda_{2}=\lambda_{3}=-\dot{\epsilon} / 2$,

- Biaxial extension: $\lambda_{1}=\lambda_{2}=\dot{\epsilon}, \lambda_{3}=-2 \dot{\epsilon}$,

- Planar extension: $\lambda_{1}=-\lambda_{2}=\dot{\epsilon}, \lambda_{3}=0$.

3 Again, the convention in the fluid mechanics literature is different than in the continuum mechanics literature, where $\mathbf{W}=\left(\mathbf{L}-\mathbf{L}^{\mathrm{T}}\right) / 2$ and is often called the spin tensor (Malvern, 1969). 


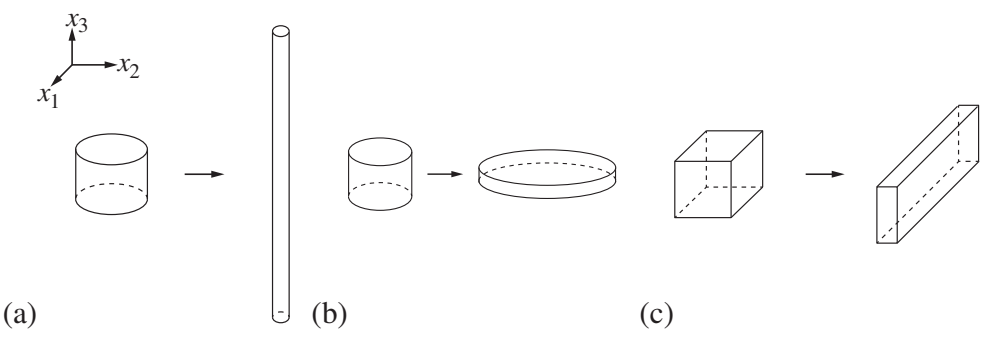

Figure 1.2 Deformation of a fluid element in (a) uniaxial extension, (b) biaxial extension, and (c) planar extension.

(a)

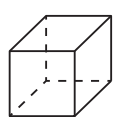

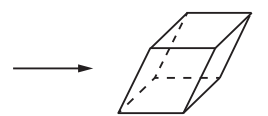

(b)

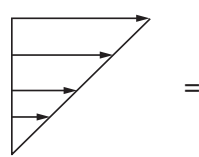

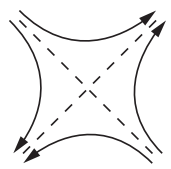

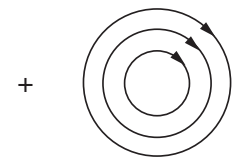

Figure 1.3 (a) Deformation of a volume of fluid in simple shear. (b) Decomposition of the velocity field of simple shear into equal parts of planar extension and rigid rotation.

In these cases, material lines will either shrink or grow exponentially in time, depending on their initial orientation relative to the eigenvectors of $\boldsymbol{\nabla} \boldsymbol{v}$. Figure 1.2 illustrates how fluid elements evolve in these flows.

Simple shear flow, where $\boldsymbol{v}=\dot{\gamma} y \boldsymbol{e}_{x}$, is another important special case that deserves particular attention. In this case, illustrated in Figure 1.3(a), elements simply move in the $x$-direction in a straight line, the eigenvalues of $\boldsymbol{\nabla} \boldsymbol{v}$ are all zero, and an arbitrarily oriented material line stretches linearly with time. In Cartesian coordinates,

$$
\boldsymbol{\nabla} \boldsymbol{v}=\left[\begin{array}{ccc}
0 & 0 & 0 \\
\dot{\gamma} & 0 & 0 \\
0 & 0 & 0
\end{array}\right]
$$

and thus

$$
\mathbf{E}=\frac{1}{2}\left[\begin{array}{lll}
0 & \dot{\gamma} & 0 \\
\dot{\gamma} & 0 & 0 \\
0 & 0 & 0
\end{array}\right]
$$

and

$$
\mathbf{W}=\frac{1}{2}\left[\begin{array}{ccc}
0 & -\dot{\gamma} & 0 \\
\dot{\gamma} & 0 & 0 \\
0 & 0 & 0
\end{array}\right]
$$

From (1.4) and (1.5),

$$
\omega=\left[\begin{array}{c}
0 \\
0 \\
-\frac{1}{2} \dot{\gamma}
\end{array}\right]
$$


(a)

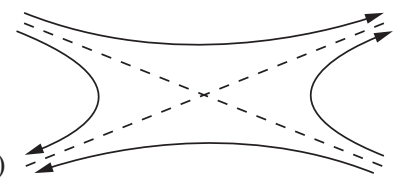

(b)

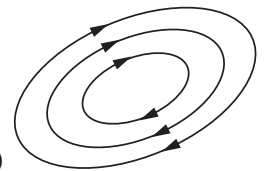

Figure 1.4 Paths of fluid elements for perturbations of simple shear with (a) $\alpha>0$ and (b) $\alpha<0$. The case $\alpha=0$ is simple shear as shown in Figure 1.3.

Letting $\|\cdot\|$ denote the Frobenius norm when applied to a second-order tensor, ${ }^{4}$ observe that in this case $\|\mathbf{E}\|=\|\mathbf{W}\|$. If the velocity gradient were composed entirely of $\mathbf{E}$, then fluid elements would undergo planar extension, compressing along the line $x=-y$, the "compressional axis" and stretching along the line $x=y$, the "extensional axis." On the other hand, if the velocity gradient were composed entirely of $\mathbf{W}$, then fluid elements would just rotate clockwise. The overall deformation that occurs during simple shear is an equal superposition of these deformations, as illustrated in Figure 1.3(b), in which the stretching due to $\mathbf{E}$ is just balanced by the rotation due to $\mathbf{W}$, and fluid elements stretch linearly in time, tilting toward the flow direction but without "tumbling." This result is a simple consequence of the fact that the particle paths are all straight lines.

Consider now a small change in the velocity gradient from the simple shear case (Fuller \& Leal 1981):

$$
\boldsymbol{\nabla} \boldsymbol{v}=\dot{\gamma}\left[\begin{array}{lll}
0 & \alpha & 0 \\
1 & 0 & 0 \\
0 & 0 & 0
\end{array}\right]
$$

Now

$$
\mathbf{E}=\frac{1}{2} \dot{\gamma}\left[\begin{array}{ccc}
0 & 1+\alpha & 0 \\
1+\alpha & 0 & 0 \\
0 & 0 & 0
\end{array}\right]
$$

and

$$
\mathbf{W}=\frac{1}{2} \dot{\gamma}\left[\begin{array}{ccc}
0 & \alpha-1 & 0 \\
1-\alpha & 0 & 0 \\
0 & 0 & 0
\end{array}\right]
$$

Holding $\dot{\gamma}$ constant and restricting $\alpha$ to the range $-1 \leq \alpha \leq 1$, there are three cases as shown in Figure 1.4. If $\alpha=0$, then the flow is simple shear. If $\alpha>0$, then strain dominates over vorticity, $\|\mathbf{E}\|>\|\mathbf{W}\|$, the eigenvalues of $\boldsymbol{\nabla} \boldsymbol{v}$ are real, particle paths are hyperbolas, and a material line stretches exponentially fast - in the limiting case $\alpha=1$, the vorticity vanishes, and flow is pure planar extension, with compressional axis along the line $x=-y$ and extensional axis along $x=y$. On the other hand, if $\alpha<0$, vorticity dominates, $\|\mathbf{E}\|<\|\mathbf{W}\|$, and particle paths are ellipses - an individual fluid element will oscillate in length. Accordingly, $\boldsymbol{\nabla} \boldsymbol{v}$ has a pair of purely imaginary eigenvalues. (There is always one zero eigenvalue, independent of $\alpha$, because there is no motion in the $z$ direction.) When $\alpha=-1$, the strain rate vanishes and the flow is rigid rotation particle paths are circles and material lines rotate without any change in length. Thus

\footnotetext{
4 See Appendix A.1.
} 


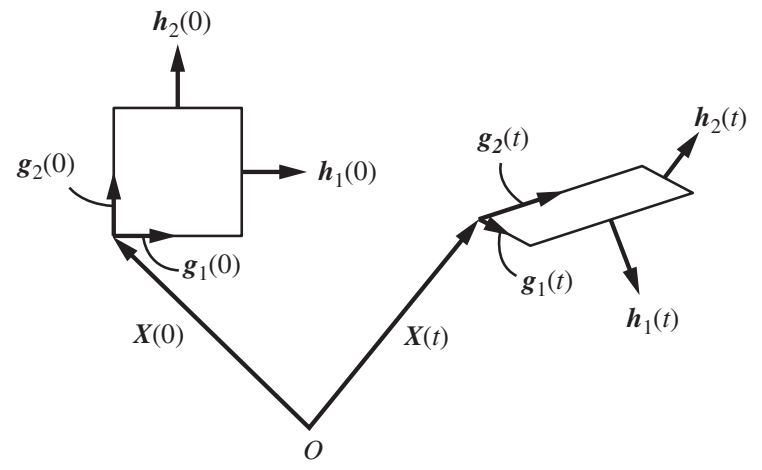

Figure 1.5 Basis vectors $\boldsymbol{g}_{i}(t)$ attached to a material point $X(t)$ and evolving as material lines. At $t=0$ (left) these vectors define the edges of a cube, only two dimensions of which are shown, that is deformed at time $t$ to a parallelepiped. The reciprocal basis vectors $\boldsymbol{h}_{i}(t)$ define the faces of the parallelepiped.

we see that simple shear is a delicate special case, a point that should be remembered because it is so often used as a model flow.

\subsubsection{Deformation Tensors}

For time-independent linear flows, the eigenvalues and eigenvectors of $\boldsymbol{\nabla} \boldsymbol{v}$ contain all the information needed to determine how material lines stretch and thus how the material deforms. For more complex flow fields, however, we require a more general formalism to characterize deformations (Malvern 1969, Bird, Armstrong, \& Hassager 1987, Segel 1987, Gonzalez \& Stuart 2008, Morozov \& Spagnolie 2015). Consider a set of basis vectors $\boldsymbol{g}_{i}$ that are attached to a material element $\boldsymbol{X}(t)$. At $t=0$, the basis vectors correspond to the Cartesian basis vectors: $\boldsymbol{g}_{i}(\boldsymbol{X}(0), 0)=\boldsymbol{e}_{i}$, where $\boldsymbol{e}_{i}$ is the $i$ th Cartesian unit basis vector. Thus the $\boldsymbol{g}_{i}$ are the edges of the parallelepiped that begins as cubic material volume attached to $\boldsymbol{X}(t)$. These basis vectors will be taken to evolve as material lines, as shown in Figure 1.5: i.e.,

$$
\frac{d \boldsymbol{g}_{i}(\boldsymbol{X}(t), t)}{d t}=\mathbf{L}(\boldsymbol{X}(t), t) \cdot \boldsymbol{g}_{i}(\boldsymbol{X}(t), t)
$$

Note that $\mathbf{L}$ is time dependent in general. These vectors form a basis that is codeforming with the material. Recall that at $t=0$, the $\boldsymbol{g}_{i}$ are tangent to the Cartesian coordinate lines. If we take these coordinate lines to be embedded in the material, moving and deforming with it, then because the $\boldsymbol{g}_{i}$ evolve as material lines, they will each be tangent to the corresponding coordinate line at time $t$; basis vectors that are parallel to coordinate lines are said to be contravariant (Aris 1989).

The solution to (1.8) can be written as

$$
\boldsymbol{g}_{i}(X(t), t)=\mathbf{F}(X(t), t) \cdot \boldsymbol{g}_{i}(X(0), 0),
$$

where $\mathbf{F}(\boldsymbol{X}(t), t)$ is simply the time-dependent mapping between $\boldsymbol{g}_{i}(0)$ and $\boldsymbol{g}_{i}(t)$ for material point $\boldsymbol{X}$. It is called the deformation gradient tensor. (For brevity of notation, we occasionally drop the dependence of the $\boldsymbol{g}_{i}$ on $\boldsymbol{X}$.) Since $\boldsymbol{g}_{i}(0)=\boldsymbol{e}_{i}$, we have that

$$
\boldsymbol{g}_{i}(t)=\mathbf{F}(X(t), t) \cdot \boldsymbol{e}_{i}
$$


or equivalently, the $i$ th column of $\mathbf{F}(\boldsymbol{X}(t), t)$ is the basis vector $\boldsymbol{g}_{i}(t): F_{j i}(\boldsymbol{X}(t), t)=$ $g_{j i}(\boldsymbol{X}(t), t)$. Inserting (1.10) into (1.8) and factoring out $\boldsymbol{e}_{i}$ yields that

$$
\frac{d \mathbf{F}}{d t}=\mathbf{L} \cdot \mathbf{F}
$$

with initial condition $\mathbf{F}(\boldsymbol{X}(0), 0)=\boldsymbol{\delta}$. In the special case of a steady linear flow, where $\mathbf{L}$ is constant, $\mathbf{F}=e^{\mathbf{L} t}$ and the $\boldsymbol{g}_{i}$ are independent of position.

Because the $\boldsymbol{g}_{i}$ evolve as material lines, Equation (1.9) also holds with $\boldsymbol{g}_{i}$ replaced with $\Delta \boldsymbol{X}$ :

$$
\Delta \boldsymbol{X}(t)=\mathbf{F}(\boldsymbol{X}(t), t) \cdot \Delta \boldsymbol{X}(0)
$$

Thus the deformation gradient tensor $\mathbf{F}$ contains all information needed to determine the evolution of a material line during a deformation. ${ }^{5}$ Recalling that the $i$ th column of $\mathbf{F}(\boldsymbol{X}(t), t)$ is the basis vector $\boldsymbol{g}_{i}(t)$, we can rewrite (1.12) in the coordinate system defined by these vectors:

$$
\Delta \boldsymbol{X}(t)=\Delta X_{1}(0) \boldsymbol{g}_{1}(t)+\Delta X_{2}(0) \boldsymbol{g}_{2}(t)+\Delta X_{3}(0) \boldsymbol{g}_{3}(t) .
$$

For $t>0$ the basis vectors $\boldsymbol{g}_{i}(t)$ are not generally orthogonal. However, it is always possible to find a reciprocal basis comprised of vectors $\boldsymbol{h}_{j}(t)$ that satisfy the so-called biorthogonality condition $\boldsymbol{g}_{i}(t) \cdot \boldsymbol{h}_{j}(t)=\delta_{i j}$. For the parallelepiped whose edges are defined by the $\boldsymbol{g}_{i}$, the normal vectors to the faces of the parallelepiped are defined by the $\boldsymbol{h}_{i}$, as shown in Figure 1.5. Now thinking of coordinate planes that are embedded in the material and evolve with it, the $\boldsymbol{h}_{i}$ are basis vectors that are orthogonal to these planes and are said to be covariant. Since the basis vectors $\boldsymbol{g}_{i}(t)$ are contained in the columns of the deformation gradient tensor $\mathbf{F}(t)$, the columns of $\left(\mathbf{F}(t)^{\mathrm{T}}\right)^{-1}$ will contain the reciprocal basis vectors $\boldsymbol{h}_{j}(t)$. These vectors satisfy

$$
\frac{d \boldsymbol{h}_{j}}{d t}=-\mathbf{L}^{\mathrm{T}} \cdot \boldsymbol{h}_{j}
$$

Problem 1.4 illustrates one context in which this reciprocal basis arises.

Just as the vectors $\boldsymbol{g}_{i}$ comprise a codeforming basis set for representing vectors associated with a deforming material, the dyads $\boldsymbol{g}_{i} \boldsymbol{g}_{j}$ form a basis for representing second-order tensors. In index notation, where $g_{k j}=\boldsymbol{e}_{k} \cdot \boldsymbol{g}_{j}$, these dyads evolve as follows:

$$
\begin{aligned}
\left(\frac{d \boldsymbol{g}_{i} \boldsymbol{g}_{j}}{d t}\right)_{l k}=\frac{d g_{l i} g_{k j}}{d t} & =\frac{d g_{l i}}{d t} g_{k j}+g_{l i} \frac{d g_{k j}}{d t} \\
& =L_{l m} g_{m i} g_{k j}+g_{l i} L_{k m} g_{m j} \\
& =L_{l m} g_{m i} g_{k j}+g_{l i} g_{m j} L_{m k}^{\mathrm{T}},
\end{aligned}
$$

which we can rewrite

$$
\frac{d \boldsymbol{g}_{i} \boldsymbol{g}_{j}}{d t}=\boldsymbol{g}_{i} \boldsymbol{g}_{j} \cdot \nabla \boldsymbol{v}+\nabla \boldsymbol{v}^{\mathrm{T}} \cdot \boldsymbol{g}_{i} \boldsymbol{g}_{j}
$$

5 In mathematical terms, $\mathbf{F}$ is the fundamental solution matrix for (1.2). 
More generally, we can define the Green tensor ${ }^{6}$

$$
\mathbf{B}(\boldsymbol{X}(t), t)=\sum_{i=1}^{3} \sum_{i=1}^{3} \boldsymbol{g}_{i} \boldsymbol{g}_{j} .
$$

By construction, at $t=0, \mathbf{B}=\delta$, where $\delta$ is the identity tensor. Using (1.15), we can write that

$$
\frac{d \mathbf{B}(X(t), t)}{d t}-\left(\mathbf{B}(\boldsymbol{X}(t), t) \cdot \boldsymbol{\nabla} \boldsymbol{v}(\boldsymbol{X}(t), t)+\boldsymbol{\nabla} \boldsymbol{v}^{\mathrm{T}}(\boldsymbol{X}(t), t) \cdot \mathbf{B}(\boldsymbol{X}(t), t)\right)=\mathbf{0} .
$$

If we think about $\mathbf{B}$ as a tensor field, i.e., as a function of position $\boldsymbol{x}$ in the flow field rather than as a tensor attached to a particular material point $\boldsymbol{X}(t)$, then the time derivative is replaced by a substantial derivative ${ }^{7}$

$$
\frac{D}{D t}=\frac{\partial}{\partial t}+\boldsymbol{v} \cdot \boldsymbol{\nabla}
$$

in which case

$$
\frac{D \mathbf{B}(x, t)}{D t}-\left(\mathbf{B}(\boldsymbol{x}, t) \cdot \boldsymbol{\nabla} \boldsymbol{v}(\boldsymbol{x}, t)+\boldsymbol{\nabla} \boldsymbol{v}^{\mathrm{T}}(\boldsymbol{x}, t) \cdot \mathbf{B}(\boldsymbol{x}, t)\right)=\mathbf{0} .
$$

The quantity on the left-hand side of this expression is called the contravariant convected derivative or upper convected derivative of $\mathbf{B}$ and is denoted $\mathbf{B}_{(1)}$ or $\stackrel{\nabla}{\mathbf{B}}$. This derivative is the rate of change of a tensor relative to a coordinate system that is deforming with the material. The Green tensor is fundamentally important to the theory of elasticity and viscoelasticity, in part because the stress tensor for a simple model of a material called the neo-Hookean solid is proportional to it. We will see this object again in Section 8.6, where it arises naturally in a model of the dynamics of dilute polymer solutions. The Green tensor is related to $\mathbf{F}$ by

$$
\mathbf{B}(\boldsymbol{X}(t), t)=\mathbf{F}(\boldsymbol{X}(t), t) \cdot \mathbf{F}^{\mathrm{T}}(\boldsymbol{X}(t), t) .
$$

(See Problem 1.2.)

6 We use the nomenclature recommended by the International Union of Pure and Applied Chemistry (IUPAC) (Kaye et al. 1998). This tensor is also called the left Cauchy-Green tensor or sometimes the Finger tensor, although according to the IUPAC standard the latter term refers to the quantity $\left(\mathbf{F}^{\mathrm{T}} \cdot \mathbf{F}\right)^{-1}$. The right Cauchy-Green tensor $\mathbf{C}$, also called the Cauchy tensor, is given by $\mathbf{C}=\mathbf{F}^{\mathrm{T}} \cdot \mathbf{F}$.

7 Consider a scalar field $f(\boldsymbol{x}(t), t)$. The rate of change of this field as measured by an observer moving with velocity $d \boldsymbol{x} / d t=\boldsymbol{v}$ (i.e., moving as a material point $\boldsymbol{X}(t)$ ) is given by the substantial derivative $D f / D t$. In Cartesian coordinates, we can use the chain rule to write this as

$$
\begin{aligned}
\frac{D f(\boldsymbol{x}, t)}{D t} & =\frac{\partial f}{\partial t}+\sum_{i=1}^{3} \frac{d x_{i}}{d t} \frac{\partial f}{\partial x_{i}} \\
& =\frac{\partial f}{\partial t}+\sum_{i=1}^{3} v_{i} \frac{\partial f}{\partial x_{i}} \\
& =\frac{\partial f}{\partial t}+\boldsymbol{v} \cdot \boldsymbol{\nabla} f .
\end{aligned}
$$



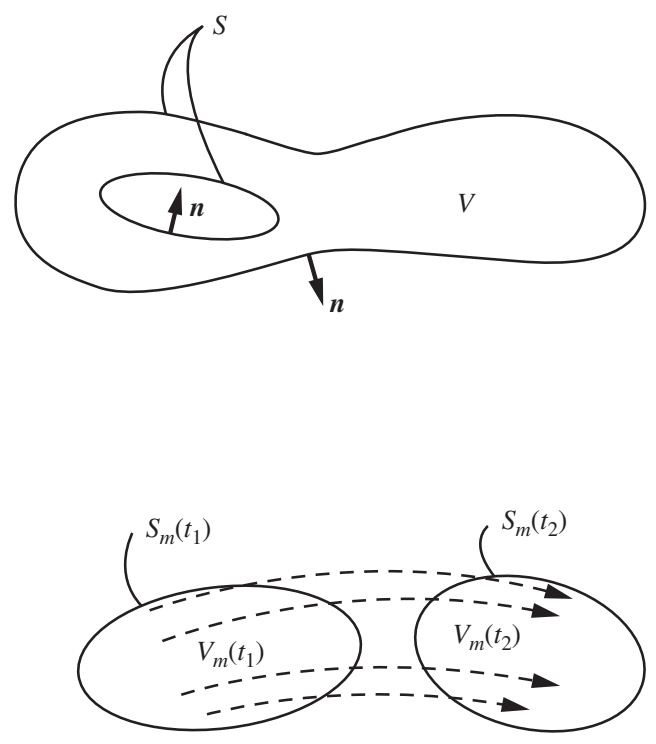

Figure 1.6 Arbitrary stationary (top) and material (bottom) volumes within a material for derivation of conservation laws.

A number of other deformation tensors and convected derivatives also arise in studies of complex fluids and elastic solids. Section 9.4 illustrates the importance of these derivatives in the general context of constitutive models for stress in a material.

\subsection{Conservation Equations}

\subsubsection{Conservation of Mass}

Consider an arbitrarily chosen stationary volume $V$ with boundary $S$ within a material of mass density $\rho$ as shown in Figure 1.6. The outward unit normal vector for the volume is denoted $\boldsymbol{n}$. Given that the mass flux at any position $\boldsymbol{x}$ in the volume is $\rho \boldsymbol{v}$, the mass balance for this domain can be written as

$$
\frac{d}{d t} \int_{V} \rho d V=-\int_{S} \boldsymbol{n} \cdot(\rho \boldsymbol{v}) d S .
$$

This equation simply states that the rate of accumulation of mass in the domain is equal to the integral over the boundary of the mass flux into the volume $-\boldsymbol{n} \cdot(\rho \boldsymbol{v})-$ mass is neither created nor destroyed within the volume $V$. Applying the divergence theorem (Equation (A.10) in Appendix A.2) to the right-hand side and rearranging yields that

$$
\frac{d}{d t} \int_{V} \rho d V+\int_{V} \boldsymbol{\nabla} \cdot(\rho \boldsymbol{v}) d V=0 .
$$

Since the volume $V$ is stationary, this can be rewritten

$$
\int_{V} \frac{\partial \rho}{\partial t} d V+\int_{V} \boldsymbol{\nabla} \cdot(\rho \boldsymbol{v}) d V=0
$$


or

$$
\int_{V}\left(\frac{\partial \rho}{\partial t}+\boldsymbol{\nabla} \cdot(\rho \boldsymbol{v})\right) d V=0 .
$$

The domain of integration is arbitrary, so the only way the integral can vanish is for the integrand to vanish pointwise:

$$
\frac{\partial \rho}{\partial t}+\nabla \cdot(\rho \boldsymbol{v})=0
$$

at every point in the domain. This is the statement of conservation of mass at a point in a continuous medium and is known as the continuity equation. We will exclusively consider incompressible flows during which density changes are negligible and the continuity equation reduces to

$$
\boldsymbol{\nabla} \cdot \boldsymbol{v}=0
$$

\subsubsection{Conservation of Momentum}

To address conservation of momentum, we take a slightly different approach than we did for mass conservation. Consider an arbitrary material volume $V_{\mathrm{M}}(t)$, by which we mean a volume whose elements move with the material velocity $\boldsymbol{v}(\boldsymbol{x}, t)$ (Figure 1.6). Therefore points on the boundary of this volume $S_{\mathrm{M}}(t)$ are also moving with this velocity. The total momentum of this volume is

$$
\int_{V_{\mathrm{M}}(t)} \rho \boldsymbol{v} d V
$$

and Newton's second law applied to it becomes

$$
\frac{d}{d t} \int_{V_{\mathrm{M}}(t)} \rho \boldsymbol{v} d V=\boldsymbol{F}_{V}+\boldsymbol{F}_{S} .
$$

Here the left-hand side is the rate of change of momentum of the volume, $\boldsymbol{F}_{V}$ incorporates forces exerted on the material in the volume by external fields that act at each point in the material and $\boldsymbol{F}_{S}$ incorporates forces exerted across the boundary of the volume by the neighboring material - stresses.

Many body forces - gravitational, electrical, magnetic - can act at each point in a material in an external field. For the moment, we do not specify the nature of these forces but simply write that

$$
\boldsymbol{F}_{V}=\int_{V_{\mathrm{M}}(t)} \boldsymbol{f}(\boldsymbol{x}) d V,
$$

where $\boldsymbol{f}(\boldsymbol{x})$ is an arbitrary position-dependent force density.

The net surface force is the integral of the stresses exerted across the boundary of $V$. Denoting the stress vector (sometimes called the traction vector) exerted at a point on the boundary by the neighboring material outside the boundary as $\boldsymbol{t}(\boldsymbol{x})$, this force can be written

$$
\boldsymbol{F}_{S}=\int_{S_{\mathrm{M}}(t)} \boldsymbol{t}(\boldsymbol{x}) d S
$$


Under very general circumstances, the traction vector has the form

$$
\boldsymbol{t}=\boldsymbol{n} \cdot \boldsymbol{\sigma},
$$

where $\sigma$ is a second-order tensor called the stress tensor (Batchelor 1967, Aris 1989, Pozrikidis 1997). In Cartesian coordinates, the quantity $\sigma_{x y}$, for example, is the force per unit area in the $y$ direction exerted on a surface of constant $x$ by the material at greater $x$. Conservation of angular momentum yields, again under very general circumstances, that the stress tensor is symmetric: $\sigma_{i j}=\sigma_{j i}$ (see Problem 1.6). The stress tensor will be the subject of further discussion later in this chapter and elsewhere in the book.

Using the expressions for $\boldsymbol{F}_{V}$ and $\boldsymbol{F}_{S}$, we can rewrite (1.24) as

$$
\frac{d}{d t} \int_{V_{\mathrm{M}}(t)} \rho \boldsymbol{v} d V=\int_{V_{\mathrm{M}}(t)} \boldsymbol{f} d V+\int_{S_{\mathrm{M}}(t)} \boldsymbol{n} \cdot \boldsymbol{\sigma} d S .
$$

Now Leibniz's rule, (A.15), can be applied to the left-hand side:

$$
\int_{V_{\mathrm{M}}(t)} \frac{\partial \rho \boldsymbol{v}}{\partial t} d V+\int_{S_{\mathrm{M}}(t)} \rho \boldsymbol{v}(\boldsymbol{n} \cdot \boldsymbol{v}) d S=\int_{V_{\mathrm{M}}(t)} \boldsymbol{f} d V+\int_{S_{\mathrm{M}}(t)} \boldsymbol{n} \cdot \boldsymbol{\sigma} d S .
$$

Combining the surface integrals yields that

$$
\int_{V_{\mathrm{M}}(t)} \frac{\partial \rho \boldsymbol{v}}{\partial t} d V=\int_{S_{\mathrm{M}}(t)} \boldsymbol{n} \cdot(-\rho \boldsymbol{v} \boldsymbol{v}+\boldsymbol{\sigma}) d S+\int_{V_{\mathrm{M}}(t)} \boldsymbol{f} d V,
$$

where we have used the definition of a dyad: $(\boldsymbol{n} \cdot \boldsymbol{v}) \boldsymbol{v}=\boldsymbol{n} \cdot(\boldsymbol{v} \boldsymbol{v})$. From this form of the equation, we can recognize that the quantity $\rho \boldsymbol{v} \boldsymbol{v}-\boldsymbol{\sigma}$ is the total momentum flux at a point in the material. The first term represents momentum carried by the bulk motion of the material and is called the convective or advective momentum flux, while the second represents momentum transported via microscopic mechanisms.

Applying the divergence theorem to the surface integral on the right-hand side converts it to a volume integral. Again, since the volume under consideration is arbitrary, we conclude that at every point in the domain the following equation holds:

$$
\frac{\partial \rho \boldsymbol{v}}{\partial t}=-\boldsymbol{\nabla} \cdot(\rho \boldsymbol{v} \boldsymbol{v})+\boldsymbol{\nabla} \cdot \boldsymbol{\sigma}+\boldsymbol{f}
$$

With some rearrangement and the use of the continuity equation, this can be written in more conventional form:

$$
\rho\left(\frac{\partial v}{\partial t}+v \cdot \nabla v\right)=\nabla \cdot \sigma+f
$$

which is called the Cauchy momentum balance equation. This equation is very broadly valid, independent of the material. Now we split the stress into an isotropic component that at equilibrium is just the thermodynamic pressure, and an additional component $\tau$, sometimes called the extra stress tensor, that vanishes at equilibrium:

$$
\sigma=-p \delta+\tau .
$$

With this definition, we have another common form of the Cauchy equation

$$
\rho\left(\frac{\partial v}{\partial t}+\boldsymbol{v} \cdot \boldsymbol{\nabla v}\right)=-\nabla p+\nabla \cdot \tau+f
$$


For an incompressible fluid, the pressure cannot be viewed as a thermodynamic quantity. It is best viewed as a Lagrange multiplier that enables the pointwise incompressibility constraint $\boldsymbol{\nabla} \cdot \boldsymbol{v}=0$ to be satisfied. Section 1.3 .4 illustrates this point explicitly for a specific case.

Microscopically, the stress tensor $\sigma$ can have many different origins. For example, it may be due to the fluctuating momentum fluxes associated with thermal motion of the molecules forming the material, or to forces exerted across the boundary due to chemical bonds that span it (Bird et al. 1987, Chaikin \& Lubensky 1995, McQuarrie 2000, Bird, Stewart \& Lightfoot 2002). Later sections of this book will shed light on the microscopic origins of the stresses associated with particles or macromolecules in solution, and we will eventually see that a complex fluid can be viscoelastic, having both a viscous contribution to the stress that is proportional to the rate of deformation and an elastic contribution that is proportional to the amplitude of deformation.

For this and the next several chapters, however, our focus will be on a specific class of materials, incompressible Newtonian fluids, in which density is constant and the contribution to the stress at a point in the fluid due to its deformation is a linear and isotropic function of the strain rate $\mathbf{E}$ at that point. The stress cannot depend on $\mathbf{W}$, because there is no material deformation associated with rotation (see Section 9.4 for further discussion). For such a fluid, the quantity $\tau$ is called the viscous stress and has the form

$$
\boldsymbol{\tau}=2 \eta \mathbf{E}=\eta\left(\boldsymbol{\nabla} \boldsymbol{v}+\boldsymbol{\nabla} \boldsymbol{v}^{\mathrm{T}}\right),
$$

where $\eta$ is the dynamic viscosity (usually just called the viscosity) of the fluid - it is the proportionality constant between stress and strain rate. The related quantity $v=\eta / \rho$, known as the kinematic viscosity, plays the role of a diffusivity for momentum transport. Now the Cauchy momentum equation reduces to the incompressible Navier-Stokes equation

$$
\rho\left(\frac{\partial v}{\partial t}+\boldsymbol{v} \cdot \nabla v\right)=-\nabla p+\eta \nabla^{2} v+f .
$$

Supplemented by (1.23) for mass conservation and appropriate boundary conditions, this equation provides a complete description of flows of incompressible Newtonian fluids. Because we only consider incompressible flow, when we write "Navier-Stokes equation" (or "Stokes equation," discussed later), we will generally mean "Navier-Stokes and continuity equations for incompressible flow."

In many situations (e.g., single-phase flow with no free interfaces, multiphase flow of density-matched fluids), the only body force is gravity and its only effect is to induce a hydrostatic pressure gradient in the fluid that has no influence on the fluid motion. Therefore, it is often useful to write the body force as the gradient of the gravitational potential per unit volume:

$$
\boldsymbol{f}=\rho \boldsymbol{g}=-\nabla \Phi_{\mathrm{g}}, \quad \Phi_{\mathrm{g}}=\rho g\left(h-h_{0}\right),
$$

where $\boldsymbol{g}$ is the gravity vector, $h$ is vertical height, and $h_{0}$ an arbitrary reference height. Now the pressure and gravitational terms can be combined by defining the dynamic 
pressure $\mathbb{P}=p+\Phi_{\mathrm{g}}$. For a fluid at rest, $\mathbb{P}$ is constant. Now the momentum equation can be written

$$
\rho\left(\frac{\partial v}{\partial t}+\boldsymbol{v} \cdot \boldsymbol{\nabla v}\right)=-\nabla \mathbb{P}+\eta \nabla^{2} \boldsymbol{v}
$$

Where there is no danger of confusion, $\mathbb{P}$ is often replaced by $p$.

Now consider a situation in which the length scale over which we expect velocities to vary is $L$ and the characteristic variation in velocities is $U$. These quantities will vary from system to system; for a particle of size $a$ in a shear flow with shear rate $\dot{\gamma}$, we could take $L=a$ and $U=a \dot{\gamma}$. Additionally, there may be a time scale $T$ for imposed variations in velocity. Using these scales, along with the viscous stress scale $\eta U / L$ as a characteristic pressure scale, we can rewrite the momentum equation in nondimensional form:

$$
\operatorname{Re}\left(\operatorname{Sr}^{-1} \frac{\partial \boldsymbol{v}}{\partial t}+\boldsymbol{v} \cdot \boldsymbol{\nabla} \boldsymbol{v}\right)=-\boldsymbol{\nabla} p+\nabla^{2} \boldsymbol{v}+\boldsymbol{f},
$$

where all variables are now nondimensionalized with their corresponding scales, and two dimensionless groups appear:

$$
\operatorname{Re}=\frac{U L}{v}
$$

and

$$
\mathrm{Sr}=\frac{T U}{L} .
$$

The Reynolds number Re estimates the ratio between the time scale for diffusion of momentum $\left(L^{2} / v\right)$ over the length scale $L$ and the time scale for flow $(L / U)$ over that scale. The Strouhal number $\mathrm{Sr}$ estimates the ratio between the imposed time scale $T$ and the flow time scale $L / U$. If there is no imposed time scale (e.g., if the flow is generated by a steady boundary motion or pressure gradient), then $T=L / U$ and $\mathrm{Sr}=1$.

For water at room temperature, $v \approx 10^{-6} \mathrm{~m}^{2} / \mathrm{s}$. If $L \approx 1 \mu \mathrm{m}$, then $\operatorname{Re} \ll 1$ as long as $U \ll 1 \mathrm{~m} / \mathrm{s}$, a condition that is satisfied for a wide variety of microscale processes. Thus, for these processes and under the condition $\mathrm{Sr}=O(1)$, the acceleration and convective terms in the Navier-Stokes equation can be neglected and the momentum balance reduces to the Stokes equation, which in dimensional form is given by

$$
-\boldsymbol{\nabla} p+\eta \nabla^{2} \boldsymbol{v}+\boldsymbol{f}=\mathbf{0} .
$$

Flow governed by the Stokes equation, or Stokes flow, is inertialess - the mass of the fluid plays no role in its dynamics. Taking the divergence of (1.35) and applying incompressibility shows that the pressure field in Stokes flow satisfies a Poisson equation:

$$
\nabla^{2} p=\nabla \cdot f .
$$

Similarly, taking the Laplacian of the Stokes equation and applying (1.36) yields that

$$
\eta \nabla^{2} \nabla^{2} v=\nabla \nabla \cdot f-\nabla^{2} f
$$

If $\boldsymbol{f}=\mathbf{0}$, this reduces to a biharmonic equation for $\boldsymbol{v}$.

Another important case arises when $\operatorname{Re} \ll 1$, but when forcing or motion at very short 
time scales is present so that $\operatorname{ReSr}^{-1}=O(1)$. In this case, the convective term in the Navier-Stokes equation is negligible but the acceleration term is not, and we arrive at the transient Stokes equation:

$$
\rho \frac{\partial \boldsymbol{v}}{\partial t}=-\nabla p+\eta \nabla^{2} \boldsymbol{v}+\boldsymbol{f} .
$$

This equation is also sometimes called the linearized Navier-Stokes equation. The Stokes and transient Stokes equations are the central equations of microscale fluid dynamics. The remainder of this chapter, as well as Chapters 2 through 4, elaborate on properties and solutions of the Stokes equations, while Chapter 5 addresses some key effects of inertia.

\subsubsection{Boundary Conditions}

Because interfacial flows are not a central topic of this book, we provide only a cursory treatment of boundary conditions at interfaces. Consider an interfacial surface $S_{I}$ that forms the boundary between two materials or phases; the unit normal vector pointing from material 1 to material 2 will be denoted $\boldsymbol{n}_{I}$, and $\left.\boldsymbol{v}\right|_{1}$ and $\left.\boldsymbol{v}\right|_{2}$ will be the velocities of the two phases at the interface. For simplicity, we will only consider interfaces at which no phase change is occurring and take the interface to be a material surface. Mass conservation requires that

$$
\left.\boldsymbol{n}_{I} \cdot \boldsymbol{v}\right|_{1}=\left.\boldsymbol{n}_{I} \cdot \boldsymbol{v}\right|_{2}
$$

at each point on the surface. This is called the no-penetration condition. This condition provides no information about the tangential velocities at the interface, but experimental observations indicate that for gases and small-molecule liquids under a wide range of conditions, the tangential velocities of the two phases are the same. This is called the no-slip boundary condition and can be written

$$
\left.\left(\boldsymbol{\delta}-\boldsymbol{n}_{I} \boldsymbol{n}_{I}\right) \cdot \boldsymbol{v}\right|_{1}=\left.\left(\boldsymbol{\delta}-\boldsymbol{n}_{I} \boldsymbol{n}_{I}\right) \cdot \boldsymbol{v}\right|_{2},
$$

where $\left(\boldsymbol{\delta}-\boldsymbol{n}_{I} \boldsymbol{n}_{I}\right)$ is the orthogonal projection operator onto the plane locally parallel to the interface. The combination of (1.39) and (1.40) implies that

$$
\left.\boldsymbol{v}\right|_{1}=\left.\boldsymbol{v}\right|_{2} .
$$

For a flat interface, or if interfacial tension effects are negligible, continuity of stress across the interface is required by the momentum balance:

$$
\left.n_{I} \cdot \sigma\right|_{1}=\left.n_{I} \cdot \sigma\right|_{2} .
$$

If, for example, material 2 is a gas with very small viscosity compared to that of material 1 , the viscous stress in the gas phase is often taken to be negligible, in which case the normal and tangential components of (1.42) simplify to

$$
\begin{gathered}
\left.p\right|_{1}=\left.p\right|_{2}, \\
\left(\boldsymbol{\delta}-\boldsymbol{n}_{I} \boldsymbol{n}_{I}\right) \cdot\left(\boldsymbol{n}_{I} \cdot \boldsymbol{\tau}\right)=\mathbf{0} .
\end{gathered}
$$


The latter equation expresses that there is no shear stress across the interface. For example, in Cartesian coordinates, if the interface is the surface $y=0$, then this condition, after application of the no-penetration condition $v_{y}=0$, becomes

$$
\frac{\partial v_{x}}{\partial y}=\frac{\partial v_{z}}{\partial y}=0
$$

For gas-liquid problems, this condition, rather than no-slip, is often applied on the liquid phase, with the motion in the gas phase simply neglected.

Finally, for an interface between two fluids with interfacial tension $\gamma$, the interfacial stress balance becomes

$$
\boldsymbol{n}_{I} \cdot\left(\left.\boldsymbol{\sigma}\right|_{2}-\left.\boldsymbol{\sigma}\right|_{1}\right)+\nabla_{S} \gamma+2 \mathcal{H} \gamma \boldsymbol{n}_{I}=\mathbf{0} .
$$

Here $\boldsymbol{\nabla}_{S}=\left(\boldsymbol{\delta}-\boldsymbol{n}_{I} \boldsymbol{n}_{I}\right) \cdot \boldsymbol{\nabla}$ is the surface gradient operator and

$$
\mathcal{H}=-\frac{1}{2} \nabla_{S} \cdot \boldsymbol{n}_{I}
$$

is the mean curvature of the interface. If fluid 1 is a bubble or drop of radius $R$ at equilibrium in a second fluid, (1.45) reduces at equilibrium to the elementary result

$$
\left.p\right|_{2}-\left.p\right|_{1}=-\frac{2 \gamma}{R}
$$

The pressure in the bubble or drop is higher than in the surrounding fluid. Leal (2007) provides a detailed discussion of transport at interfaces.

\subsection{General Properties of Stokes Flow}

The Stokes equation (1.35) displays a number of important general properties that follow from the absence of inertial effects in the low Reynolds number or Stokes flow regime. These properties arise repeatly as we analyze and predict the dynamics of small-scale flow so we introduce them here.

\subsubsection{Linearity and Reversibility}

Consider flow in some domain $V_{\mathrm{D}}$ with boundary conditions applied on $S_{\mathrm{D}}$. Leaving aside for the moment the issue of boundary conditions, we observe that this system of equations is linear, so it is straightforward to verify by substitution into the equations the following properties:

1. Let $\boldsymbol{f}=0$. If $\left(\boldsymbol{v}_{1}, p_{1}\right)$ and $\left(\boldsymbol{v}_{2}, p_{2}\right)$ are both solutions, then so is $\left(\alpha \boldsymbol{v}_{1}+\beta \boldsymbol{v}_{2}, \alpha p_{1}+\beta p_{2}\right)$, where $\alpha$ and $\beta$ are arbitrary constant scalars.

2. Now consider the case $\boldsymbol{f} \neq 0$. If $\left(\boldsymbol{v}_{1}, p_{1}\right)$ is a solution for $\boldsymbol{f}=\boldsymbol{f}_{1}$ and $\left(\boldsymbol{v}_{2}, p_{2}\right)$ is a solution for $\boldsymbol{f}=\boldsymbol{f}_{2}$, then $\left(\alpha \boldsymbol{v}_{1}+\beta \boldsymbol{v}_{2}, \alpha p_{1}+\beta p_{2}\right)$ is a solution for $\boldsymbol{f}=\alpha \boldsymbol{f}_{1}+\beta \boldsymbol{f}_{2}$, again where $\alpha$ and $\beta$ are arbitrary constant scalars. 


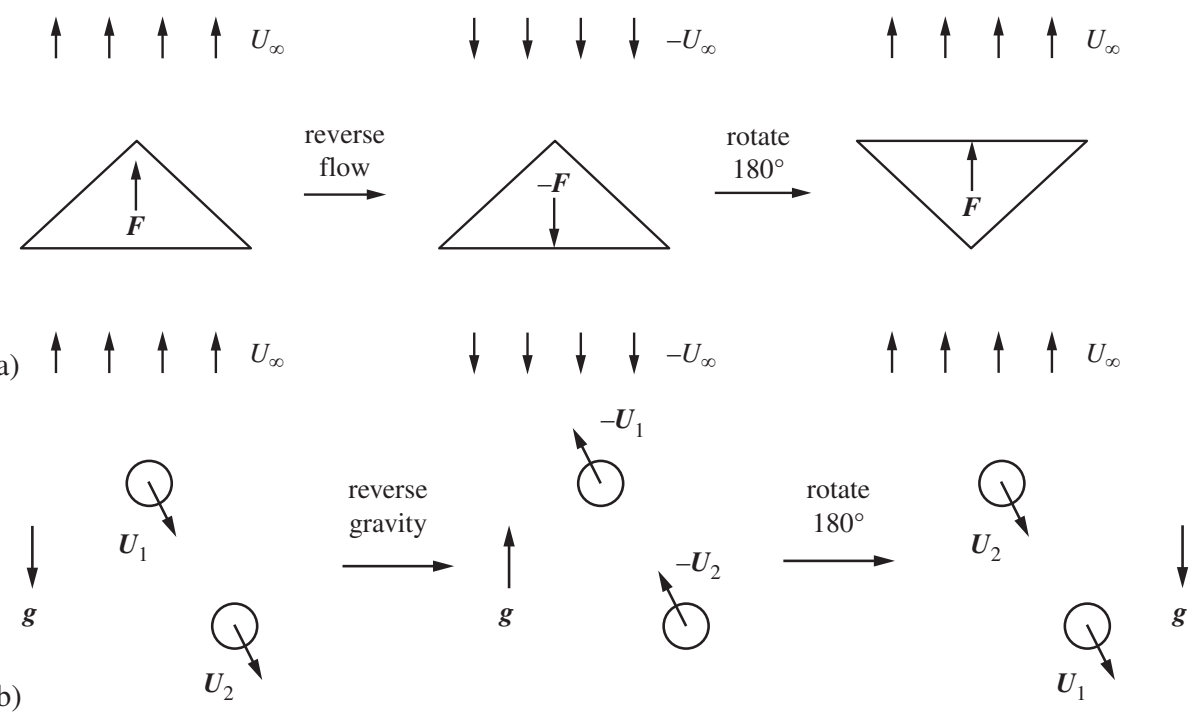

Figure 1.7 (a) Drag on an object with triangular cross-section. (b) Sedimenting pair of identical rigid spheres.

Now turning to the issue of boundary conditions, for definiteness let us consider a no-slip condition

$$
\boldsymbol{v}=\boldsymbol{v}_{\mathrm{D}} \quad \text { for } \boldsymbol{x} \in S_{\mathrm{D}}
$$

where $\boldsymbol{v}_{\mathrm{D}}$ is the specified velocity of points on the boundary - e.g., $\boldsymbol{v}_{\mathrm{D}}=0$ on a stationary wall. Again, linearity allows us to state the following result:

3. Let $\boldsymbol{f}=0$. If $\left(\boldsymbol{v}_{1}, p_{1}\right)$ is a solution for $\boldsymbol{v}_{\mathrm{D}}=\boldsymbol{v}_{\mathrm{D} 1}$ and $\left(\boldsymbol{v}_{2}, p_{2}\right)$ is a solution for $\boldsymbol{v}_{\mathrm{D}}=\boldsymbol{v}_{\mathrm{D} 2}$, then $\left(\alpha \boldsymbol{v}_{1}+\beta \boldsymbol{v}_{2}, \alpha p_{1}+\beta p_{2}\right)$ is a solution for $\boldsymbol{v}_{\mathrm{D}}=\alpha \boldsymbol{v}_{\mathrm{D} 1}+\beta \boldsymbol{v}_{\mathrm{D} 2}$.

These properties are all specific cases of the superposition principle for linear equations. They will be very important as we construct solutions to the Stokes equation, as well as in deriving the reversibility property that we describe now.

From linearity it is simple to see the following:

1. In a given domain, if $\boldsymbol{v}_{1}$ is a flow driven by forcing $\boldsymbol{f}_{1}$, then the forcing $-\boldsymbol{f}_{1}$ drives the velocity $-v_{1}$. I.e., the flow reverses.

2. Similarly, for $\boldsymbol{f}=0$, if $\boldsymbol{v}_{1}$ is a flow driven by boundary motion $\boldsymbol{v}_{\mathrm{D} 1}$, then the boundary motion $-\boldsymbol{v}_{\mathrm{D} 1}$ drives the velocity $-\boldsymbol{v}_{1}$.

These simple statements summarize the reversibility principle for Stokes flow. Especially in conjunction with symmetries of the flow and geometry, this principle can be used to 

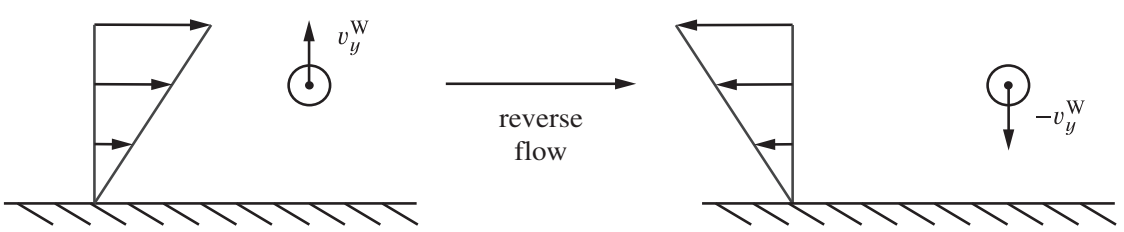

Figure 1.8 Rigid sphere in shear flow near a plane rigid surface. By reversibility, the particle velocity in the right figure must be the negative of that in the left.

draw important qualitative conclusions about forces and motions involving Stokes flow. Here are some examples.

\section{Drag on an Object.}

Consider flow around a rigid object whose cross-section is a left-right symmetric isosceles triangle as shown in Figure 1.7(a). How does the drag change if we turn the object upside down? Consider first the leftmost drawing. The object is held stationary against a drag force $\boldsymbol{F}_{\text {drag }}$ in a flow that has the uniform velocity $\boldsymbol{v}_{\infty}=U_{\infty} \boldsymbol{e}_{z}$ far from the object. By reversibility, if we change the sign of $\boldsymbol{v}_{\infty}$, as shown in the center figure, the entire velocity field will change sign, as will the pressure field, and accordingly the drag force $\boldsymbol{F}_{\text {drag }}$ will change sign. Now note that the right figure is equivalent to the center figure viewed upside down and thus equivalent to the left figure except with the object turned upside down. Therefore, we can can conclude that in Stokes flow, the drag force on this object is the same when the object is upside down as when it is upright.

\section{Sedimentation of a Pair of Rigid Spheres.}

The left panel of Figure 1.7(b) shows a pair of identical rigid spheres sedimenting due to gravity with velocities $\boldsymbol{U}_{1}$ and $\boldsymbol{U}_{2}$ in an unbounded flow. Will these particles move relative to one another as they fall? If the sign of gravity is reversed $(\boldsymbol{g} \rightarrow-\boldsymbol{g})$, then Stokes flow reversibility implies that $\boldsymbol{U}_{1} \rightarrow-\boldsymbol{U}_{1}$ and $\boldsymbol{U}_{2} \rightarrow-\boldsymbol{U}_{2}$ as shown in the center panel. Now, rotating the entire system around the midpoint between the two particles yields the right panel. This configuration is identical to the left panel but with $\boldsymbol{U}_{1}$ replaced by $\boldsymbol{U}_{2}$ and vice versa. Therefore, $\boldsymbol{U}_{1}$ and $\boldsymbol{U}_{2}$ must be identical: the particles exhibit no relative motion as they sediment. However, if an initially collinear arrangement of three or more particles is considered, there is insufficient symmetry in the problem to conclude that the particles all fall at the same velocity and in fact they will not (Problem 2.5).

\section{Motion of a Rigid Sphere near a Wall.}

We commonly encounter situations in which a particle is in a flow near a rigid wall, as shown in Figure 1.8. Will a rigid sphere migrate in Stokes flow? Assume that in the flow shown on the left, the particle migrates at wall-normal speed $v_{y}^{\mathrm{W}}$. In Stokes flow, when we reverse the imposed velocity field as shown on the right, the wall-normal velocity must become $v_{y}^{\mathrm{W}^{\prime}}=-v_{y}^{\mathrm{W}}$ (so if the particle in the left figure is moving away from the wall, the particle in the right figure is moving toward it). But by symmetry, the left and right figures are equivalent (they are two views of the same process), so $v_{y}^{\mathrm{W}^{\prime}}=v_{y}^{\mathrm{W}}$. The 
(a)

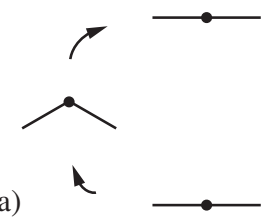

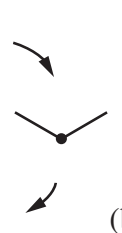

(b)

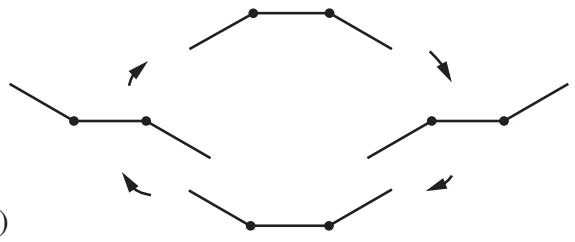

Figure 1.9 (a) Cyclic motion of two rods connected by a motorized hinge. (b) Cyclic motion of three rods connected at two hinges.

only way for $v_{y}^{\mathrm{W}}$ to satisfy both reversibility and symmetry is for it to be zero. By a very similar argument, a sphere sedimenting near to a vertical rigid wall in Stokes flow moves parallel to the wall. This argument relies both on reversibility and symmetry, the latter of which is lost if the particle is not spherical. Furthermore, due to deformability or inertial effects, particles that are spherical at rest can migrate toward or away from walls during flow, as we will describe in Sections 4.4 and 5.4.

\section{Locomotion of Linked Rigid Bodies.}

Consider the two rods connected by a hinge shown. The hinge is "motorized" so that it moves the rods relative to one another in a cyclic manner as shown in Figure 1.9(a). Can this motion generate locomotion in Stokes flow? Consider the cycle starting from the top figure in which the rods are aligned. Moving clockwise, the rods first fold upward toward each other, then back down, passing through the initial conformation as they fold downward. Then they fold back up to the straight initial conformation, completing one cycle of motion. In Stokes flow, any net motion of the object as the rods move upward away from the initial straight conformation will be exactly undone when the rods move back down into the initial configuration and likewise when the rods move down. Since in Stokes flow the fluid velocity is linearly proportional to the boundary velocity, this result holds even if the rods are moved quickly upward and slowly downward as long as both motions are slow enough that the inertia of the fluid remains negligible. More generally, reciprocal motions of boundaries, that is, cyclic motions that appear the same running forward or backward in time, lead to no net motion in Stokes flow (Purcell 1977, Lauga $\&$ Powers 2009). On the other hand, for the three-rod object undergoing the sequence of motions shown in Figure 1.9(b), no part of this cycle reverses the motion of the object induced by an earlier part. With this nonreciprocal motion, Stokes flow reversibility does not preclude net motion after one cycle. Indeed, this object will display a net translation through the fluid, a crude form of locomotion.

\subsubsection{Stress Equilibrium}

We noted earlier that at low Reynolds number, the transport of momentum by viscous diffusion is very rapid compared to transport by convection. An important consequence of this fact is known as stress equilibrium. To elucidate this mathematically, we observe that in the absence of body forces, the Stokes equation (1.35) is equivalent to the 
statement

$$
\boldsymbol{\nabla} \cdot \boldsymbol{\sigma}=\mathbf{0}
$$

(The Stokes equation in its usual form is recovered by inserting the Newtonian constitutive equation $\sigma=-p \delta+2 \eta \mathbf{E}$.) Integrating this equation over an arbitrary volume $V$ in the fluid and then applying the divergence theorem yields that

$$
\int_{S} \boldsymbol{n} \cdot \boldsymbol{\sigma} d S=\int_{S} \boldsymbol{t} d S=\boldsymbol{F}_{S}=0
$$

That is, the sum of forces on the boundary of any domain in the fluid is always zero the stresses are in equilibrium. If body forces are present, then

$$
\nabla \cdot \sigma+f=0
$$

and repeating the preceding steps yields that

$$
\int_{S} t d S+\int_{V} f d V=0 .
$$

The sum of the stresses and the body forces is zero: any body forces exerted within the fluid are instantaneously transmitted to the boundary or, if the domain is unbounded, to infinity. This point will be revisited in Section 5.1.

\subsubsection{Lorentz Reciprocal Relations}

The divergence theorem provides an important link between behavior in the interior of a domain and at its boundaries. Generalizations of this theorem provide such relationships for multiple functions. For example, given two scalar fields $\phi^{\prime}$ and $\phi^{\prime \prime}$, Green's second identity, (A.17), states that

$$
\int_{V}\left(\phi^{\prime} \nabla^{2} \phi^{\prime \prime}-\phi^{\prime \prime} \nabla^{2} \phi^{\prime}\right) d V=\int_{S}\left(\phi^{\prime} \boldsymbol{n} \cdot \boldsymbol{\nabla} \phi^{\prime \prime}-\phi^{\prime \prime} \boldsymbol{n} \cdot \boldsymbol{\nabla} \phi^{\prime}\right) d S .
$$

Letting $\boldsymbol{u}=-\boldsymbol{\nabla} \phi$, this becomes

$$
\int_{V}\left(\phi^{\prime} \boldsymbol{\nabla} \cdot \boldsymbol{u}^{\prime \prime}-\phi^{\prime \prime} \nabla \cdot \boldsymbol{u}^{\prime}\right) d V=\int_{S}\left(\phi^{\prime} \boldsymbol{n} \cdot \boldsymbol{u}^{\prime \prime}-\phi^{\prime \prime} \boldsymbol{n} \cdot \boldsymbol{u}^{\prime}\right) d S .
$$

If both $\phi^{\prime}$ and $\phi^{\prime \prime}$ are solutions to Laplace's equation, $\nabla^{2} \phi=0$, then this identity gives us a relationship between $\phi^{\prime}$ and $\phi^{\prime \prime}$ :

$$
\int_{S}\left(\phi^{\prime} \boldsymbol{n} \cdot \boldsymbol{u}^{\prime \prime}-\phi^{\prime \prime} \boldsymbol{n} \cdot \boldsymbol{u}^{\prime}\right) d S=0 .
$$

An analogous set of results applies to the Stokes equation. Consider velocity fields $\boldsymbol{v}^{\prime}$ and $\boldsymbol{v}^{\prime \prime}$ and their corresponding stress tensors $\sigma^{\prime}$ and $\sigma^{\prime \prime}$. Beginning with an expression analogous to the left-hand side of (1.52), we can use the product rule and divergence 
theorem to show that ${ }^{8}$

$$
\begin{aligned}
\int_{V}\left(\boldsymbol{v}^{\prime} \cdot\left(\boldsymbol{\nabla} \cdot \boldsymbol{\sigma}^{\prime \prime}\right)-\boldsymbol{v}^{\prime \prime} \cdot\left(\boldsymbol{\nabla} \cdot \boldsymbol{\sigma}^{\prime}\right)\right) d V= & \int_{S}\left(\boldsymbol{v}^{\prime} \cdot\left(\boldsymbol{n} \cdot \boldsymbol{\sigma}^{\prime \prime}\right)-\boldsymbol{v}^{\prime \prime} \cdot\left(\boldsymbol{n} \cdot \boldsymbol{\sigma}^{\prime}\right)\right) d S \\
& -\int_{V}\left(\boldsymbol{\nabla} \boldsymbol{v}^{\prime}: \boldsymbol{\sigma}^{\prime \prime}-\boldsymbol{\nabla} \boldsymbol{v}^{\prime \prime}: \boldsymbol{\sigma}^{\prime}\right) d S .
\end{aligned}
$$

Inserting the Newtonian form of the stress tensor, (1.28), the last term on the right-hand side can be written

$$
\begin{aligned}
& \int_{V}\left(\boldsymbol{\nabla} \boldsymbol{v}^{\prime}: \boldsymbol{\sigma}^{\prime \prime}-\boldsymbol{\nabla} \boldsymbol{v}^{\prime \prime}: \boldsymbol{\sigma}^{\prime}\right) d V \\
& \quad=\int_{V}\left(\boldsymbol{\nabla} \boldsymbol{v}^{\prime}:\left(-p^{\prime \prime} \boldsymbol{\delta}+\eta\left(\boldsymbol{\nabla} \boldsymbol{v}^{\prime \prime}+\boldsymbol{\nabla} \boldsymbol{v}^{\prime \prime \mathrm{T}}\right)\right)-\boldsymbol{\nabla} \boldsymbol{v}^{\prime \prime}:\left(-p^{\prime} \boldsymbol{\delta}+\eta\left(\boldsymbol{\nabla} \boldsymbol{v}^{\prime}+\boldsymbol{\nabla} \boldsymbol{v}^{\prime \mathrm{T}}\right)\right) d V .\right.
\end{aligned}
$$

The terms containing pressure vanish, because $\boldsymbol{\nabla} \boldsymbol{v}: \boldsymbol{\delta}=\boldsymbol{\nabla} \cdot \boldsymbol{v}=0$, and the other terms cancel because $\boldsymbol{\nabla} \boldsymbol{v}^{\prime}: \boldsymbol{\nabla} \boldsymbol{v}^{\prime \prime T}=\boldsymbol{\nabla} \boldsymbol{v}^{\prime T}: \boldsymbol{\nabla} \boldsymbol{v}^{\prime \prime}$. Therefore, this entire integral vanishes, reducing (1.54) to

$$
\int_{V}\left(\boldsymbol{v}^{\prime} \cdot\left(\boldsymbol{\nabla} \cdot \boldsymbol{\sigma}^{\prime \prime}\right)-\boldsymbol{v}^{\prime \prime} \cdot\left(\boldsymbol{\nabla} \cdot \boldsymbol{\sigma}^{\prime}\right)\right) d V=\int_{S}\left(\boldsymbol{v}^{\prime} \cdot\left(\boldsymbol{n} \cdot \boldsymbol{\sigma}^{\prime \prime}\right)-\boldsymbol{v}^{\prime \prime} \cdot\left(\boldsymbol{n} \cdot \boldsymbol{\sigma}^{\prime}\right)\right) d S .
$$

This is the most fundamental and general of the Lorentz reciprocal relations; it is valid for any incompressible Newtonian flow. The other Lorentz reciprocal relations, which we present now, are special cases of (1.55) for solutions of the Stokes equation with no imposed body force: $\boldsymbol{\nabla} \cdot \boldsymbol{\sigma}^{\prime}=\boldsymbol{\nabla} \cdot \boldsymbol{\sigma}^{\prime \prime}=0$. Now the left-hand side of (1.55) vanishes, so

$$
\int_{S}\left(\boldsymbol{v}^{\prime} \cdot\left(\boldsymbol{n} \cdot \boldsymbol{\sigma}^{\prime \prime}\right)-\boldsymbol{v}^{\prime \prime} \cdot\left(\boldsymbol{n} \cdot \boldsymbol{\sigma}^{\prime}\right)\right) d S=0 .
$$

The quantity $\boldsymbol{n} \cdot \boldsymbol{\sigma}$ is the traction vector $\boldsymbol{t}$, so (1.56) can be rewritten

$$
\int_{S}\left(\boldsymbol{v}^{\prime} \cdot \boldsymbol{t}^{\prime \prime}-\boldsymbol{v}^{\prime \prime} \cdot \boldsymbol{t}^{\prime}\right) d S=0
$$

Applying the divergence theorem to (1.56) leads to the volumetric form

$$
\int_{S}\left(\boldsymbol{\nabla} \cdot\left(\boldsymbol{\sigma}^{\prime \prime} \cdot \boldsymbol{v}^{\prime}\right)-\boldsymbol{\nabla} \cdot\left(\boldsymbol{\sigma}^{\prime} \cdot \boldsymbol{v}^{\prime \prime}\right)\right) d V=0 .
$$

Equations (1.55)-(1.58) are widely used in situations where the primed problem has a known solution and we seek information about a related problem, which is taken to be the double-primed problem. Examples of such applications are found in Sections 3.5, 4.1, 4.6, and 6.4.

\subsubsection{Mechanical Energy Balance and the Minimum Dissipation Principle}

Many examples can be found in which physical laws can be stated as variational principles. For example, thermodynamic equilibrium is the state that maximizes entropy subject to various constraints (Robertson 1993), and Newton's equations of motion can be derived from Hamilton's principle of least action (Goldstein 1980). In the context of

\footnotetext{
8 Equation (A.4) defines the double dot product as used in this book.
} 
fluid dynamics, a natural question to ask is, "what is the flow that minimizes the energy dissipation rate subject to the constraint of incompressibility?"

To address this question, we first consider the mechanical energy balance for an incompressible continuum, obtained by taking the dot product of the Cauchy momentum equation with the velocity $\boldsymbol{v}$ and rearranging:

$$
\frac{D}{D t}\left(\frac{1}{2} \rho v^{2}\right)=\boldsymbol{v} \cdot \boldsymbol{\nabla} \cdot \boldsymbol{\sigma}+\boldsymbol{v} \cdot \boldsymbol{f}
$$

Here $v^{2}=\boldsymbol{v} \cdot \boldsymbol{v}$. The left-hand side of this expression is simply the rate of change of kinetic energy of a material element. Integrating over the entire domain $V_{\mathrm{D}}$ of flow and applying the divergence theorem, we find that

$$
\int_{V_{\mathrm{D}}} \frac{D}{D t}\left(\frac{1}{2} \rho v^{2}\right) d V=\int_{S_{\mathrm{D}}} \boldsymbol{n} \cdot \boldsymbol{\sigma} \cdot \boldsymbol{v} d S+\int_{V_{\mathrm{D}}} \boldsymbol{v} \cdot \boldsymbol{f} d V-\Phi_{v},
$$

where

$$
\Phi_{v}=\int_{V_{\mathrm{D}}} \sigma: \nabla v d V
$$

The left-hand side of (1.60) is the total rate of change of kinetic energy of the material. The first two terms on the right-hand side are the rates of work on the material exerted across the domain boundary and via the body forces within the material. The last term is the one of primary interest here: it is the rate of interconversion between kinetic and internal energy (Bird et al. 2002). For an incompressible Newtonian fluid, the expression for $\Phi_{v}$ reduces to

$$
\Phi_{v}=\int_{V} \boldsymbol{\tau}: \boldsymbol{\nabla} \boldsymbol{v} d V=\int_{V} 2 \eta \mathbf{E}: \boldsymbol{\nabla} \boldsymbol{v} d V=\int_{V} \eta\left(\boldsymbol{\nabla} \boldsymbol{v}+\boldsymbol{\nabla} \boldsymbol{v}^{\mathrm{T}}\right): \boldsymbol{\nabla} \boldsymbol{v} d V
$$

and it is straightforward to show that this is always nonnegative. (For a material with some elasticity, $\Phi_{v}$ can be negative, in which case stored elastic energy is being converted to mechanical energy.)

To address the previously posed question, we wish to minimize $\Phi_{v}$ subject to the incompressibility constraint, and we will consider the case of a given velocity on the boundary. Since incompressibility must apply at every point in the fluid, the Lagrange multiplier associated with that constraint must be a function of position. Denoting this as $\lambda(\boldsymbol{x})$, we write the objective functional to be extremized as

$$
I=\int_{V}\left(\eta\left(\boldsymbol{\nabla} \boldsymbol{v}+\boldsymbol{\nabla} \boldsymbol{v}^{\mathrm{T}}\right): \boldsymbol{\nabla} \boldsymbol{v}-\lambda(\boldsymbol{x}) \boldsymbol{\nabla} \cdot \boldsymbol{v}\right) d V,
$$

subject to incompressibility and the boundary condition

$$
\boldsymbol{v}=\boldsymbol{v}_{\mathrm{D}}
$$

on the boundary $S_{\mathrm{D}}$. This is a standard application of the calculus of variations (Greenberg 1978).

It will be convenient to work in index notation. We define a comparison function

$$
V_{i}=v_{i}+\epsilon w_{i},
$$


where $w_{i}=0$ on the boundary but is otherwise arbitrary, and $v_{i}$ is the velocity field that yields the extremum. Since $w_{i}=0$ on the boundary, $V_{i}=v_{i}=v_{\mathrm{D} i}$ there. We seek the equation that must be satisfied by $v_{i}$ such that

$$
I(\epsilon)=\int_{V}\left(\eta\left(\frac{\partial V_{i}}{\partial x_{j}}+\frac{\partial V_{j}}{\partial x_{i}}\right) \frac{\partial V_{i}}{\partial x_{j}}-\lambda(\boldsymbol{x}) \frac{\partial V_{i}}{\partial x_{i}}\right) d V
$$

is an extremum. This occurs when

$$
\left.\frac{d I}{d \epsilon}\right|_{\epsilon=0}=0 .
$$

Substituting in the definition of $V_{i}$, this equation becomes

$$
\int_{V}\left[2 \eta\left(\frac{\partial v_{i}}{\partial x_{j}}+\frac{\partial v_{j}}{\partial x_{i}}\right) \frac{\partial w_{i}}{\partial x_{j}}-\lambda \frac{\partial w_{i}}{\partial x_{i}}\right] d V=0 .
$$

The first term can be written using the product rule:

$$
\int_{V}\left[\frac{\partial}{\partial x_{j}}\left(\left(\frac{\partial v_{j}}{\partial x_{i}}+\frac{\partial v_{i}}{\partial x_{j}}\right) w_{i}\right)-w_{i}\left(\frac{\partial}{\partial x_{j}}\left(\frac{\partial v_{j}}{\partial x_{i}}+\frac{\partial v_{i}}{\partial x_{j}}\right)\right)\right] d V,
$$

and applying the divergence theorem yields:

$$
\int_{S} n_{j}\left(\frac{\partial v_{j}}{\partial x_{i}}+\frac{\partial v_{i}}{\partial x_{j}}\right) w_{i} d S-\int_{V} w_{j}\left(\frac{\partial}{\partial x_{j}}\left(\frac{\partial v_{j}}{\partial x_{i}}+\frac{\partial v_{i}}{\partial x_{j}}\right)\right) d V .
$$

Similarly, the second term becomes

$$
-\int_{S} n_{j} w_{j} \lambda d S+\int_{V} w_{j} \frac{\partial \lambda}{\partial x_{j}} d V
$$

The boundary terms vanish because $w_{i}=0$ there, so substituting these expressions into the extremality condition and applying incompressibility yields that

$$
\int_{V}\left[-2 \eta \frac{\partial^{2} v_{i}}{\partial x_{j}^{2}}+\frac{\partial \lambda}{\partial x_{i}}\right] w_{i} d V=0
$$

Since $w_{i}$ is arbitrary, the term in the square brackets must be zero everywhere in the domain. Letting $\lambda=2 p$, we therefore have that

$$
=\eta \frac{\partial^{2} v_{i}}{\partial x_{j}^{2}}-\frac{\partial p}{\partial x_{i}}=0 .
$$

This is the Stokes equation, with pressure appearing as the Lagrange multiplier needed to satisfy incompressibility. Finally, to show that the solution to the Stokes equation yields the minimum dissipation, it suffices to show that any other velocity field that satisfies the boundary conditions has greater dissipation (Problem 1.8). Thus the minimum dissipation principle states that the incompressible Newtonian flow satisfying the boundary condition (1.64) that has the least dissipation is the solution to the Stokes flow problem in that geometry.

It is important to note that this minimum dissipation result does not imply, for example, that a flowing particle suspension minimizes dissipation, because in that problem the boundaries are moving with time and the preceding statement applies only to an instant 
of time - the aforementioned minimization statement does not incorporate the timedependent motion of the particles, so it says nothing about the suspension dynamics as a whole.

\section{Problems}

1.1 Find the eigenvalues and eigenvectors of (1.7) for arbitrary $\alpha \in[-1,1]$ and use the results to show that Figure 1.4 is correct.

1.2 Let $\boldsymbol{A}(\boldsymbol{X}(t), t)=\mathbf{F}(\boldsymbol{X}(t), t) \cdot \mathbf{F}(\boldsymbol{X}(t), t)^{\mathrm{T}}$. Show that

$$
\frac{d \boldsymbol{A}(\boldsymbol{X}(t), t)}{d t}=\left(\boldsymbol{A}(\boldsymbol{X}(t), t) \cdot \boldsymbol{\nabla} \boldsymbol{v}(\boldsymbol{X}(t), t)+\boldsymbol{\nabla} \boldsymbol{v}^{\mathrm{T}}(\boldsymbol{X}(t), t) \cdot \boldsymbol{A}(\boldsymbol{X}(t), t)\right) .
$$

Since $\boldsymbol{A}(\boldsymbol{X}(0), 0)=\mathbf{F}(\boldsymbol{X}(0), 0) \cdot \mathbf{F}(\boldsymbol{X}(0), 0)^{\mathrm{T}}=\boldsymbol{\delta}=\mathbf{B}(\boldsymbol{X}(0), 0)$, this is equivalent to deriving (1.19).

1.3 The inverse $\mathbf{B}^{-1}$ of the Green tensor is called the Piola tensor. Show that the evolution equation for $\mathbf{B}^{-1}$, which defines the lower or covariant convected derivative, is given by

$$
\frac{D \mathbf{B}^{-1}(\boldsymbol{x}, t)}{D t}+\left(\mathbf{B}^{-1}(\boldsymbol{x}, t) \cdot \boldsymbol{\nabla} \boldsymbol{v}^{\mathrm{T}}(\boldsymbol{x}, t)+\boldsymbol{\nabla} \boldsymbol{v}(\boldsymbol{x}, t) \cdot \mathbf{B}^{-1}(\boldsymbol{x}, t)\right)=\left(\mathbf{B}^{-1}\right)^{(1)}=\stackrel{\Delta}{\Delta}=\mathbf{B}^{-1}=0 .
$$

Hint: $D\left(\mathbf{B} \cdot \mathbf{B}^{-1}\right) / D t=0$.

1.4 In the absence of molecular diffusion, the evolution of the concentration $c(x, t)$ of a solute in an incompressible fluid is given by

$$
\frac{\partial c}{\partial t}+\boldsymbol{v} \cdot \boldsymbol{\nabla} c=0
$$

Consider a linear flow field $\boldsymbol{v}=\mathbf{L} \cdot \boldsymbol{x}$ and an initial concentration field that varies sinusoidally with position, so it can be written as $c(x, 0)=c_{0} e^{i \boldsymbol{k} \cdot \boldsymbol{x}}+$ c.c. Seeking a solution $c(\boldsymbol{x}, t)=c_{0} e^{i \boldsymbol{k}(t) \cdot \boldsymbol{x}}+$ c.c., find the evolution equation for the time-dependent wavevector $\boldsymbol{k}(t)$. How is this vector related to $\boldsymbol{g}_{i}(t)$ and/or $\boldsymbol{h}_{j}(t)$ ?

1.5 (a) For the general shear flow case (no eigenvalues of $\mathbf{L}$ with positive real parts), we can write

$$
\mathbf{L}=\left[\begin{array}{lll}
0 & a & 0 \\
0 & 0 & b \\
0 & 0 & 0
\end{array}\right]
$$

If $a$ and $b$ are constant, how fast can a material line stretch in this flow?

(b) Now, allowing a general time-dependent velocity gradient, use (1.11) to find an expression for the time averaged stretch rate

$$
\sigma=\lim _{t \rightarrow \infty} \frac{1}{t} \ln \left(\frac{\|\Delta X(t)\|}{\|\Delta X(0)\|}\right)
$$

in terms of $\mathbf{F}(t)$ and $\Delta \boldsymbol{X}(0)$. If $\sigma>0$ for some initial material line orientation $\Delta \boldsymbol{X}(0)$, then the material line stretches (and nearby fluid elements diverge) exponentially fast, on average. For each trajectory in a flow, there are three independent values of $\sigma$; they are called Lyapunov exponents. 
(c) Find the Lyapunov exponents for uniaxial extensional flow with extension rate $\dot{\epsilon}$, for which there is an orthogonal coordinate system in which

$$
\mathbf{L}=\dot{\epsilon}\left[\begin{array}{ccc}
1 & 0 & 0 \\
0 & -\frac{1}{2} & 0 \\
0 & 0 & -\frac{1}{2}
\end{array}\right]
$$

(d) The end-to-end vector $\boldsymbol{Q}$ for a simple model of polymer molecule in flow (Section 8.6) satisfies

$$
\frac{d \boldsymbol{Q}}{d t}=\mathbf{L} \cdot \boldsymbol{Q}-\frac{2}{\lambda} \boldsymbol{Q}(t)+\boldsymbol{\xi}(t),
$$

where $\lambda$ is a relaxation time for the polymer and $\boldsymbol{\xi}(t)$ is a rapidly fluctuating term that comes from Brownian motion (Chapter 6). Neglecting this term (which does not affect the final result), find a criterion relating $\sigma$ and $\lambda$ that determines whether or not a polymer molecule will stretch indefinitely along a trajectory. Hint: Use an integrating factor.

1.6 The angular momentum of a material volume $V_{\mathrm{M}}$ with respect to some fixed origin is

$$
\int_{V_{\mathrm{M}}} \boldsymbol{x} \times(\rho \boldsymbol{v}) d V
$$

If we allow external torques to be imposed at points within a material (this could occur, for example, by application of an external electric field to a fluid of polar molecules), then the conservation of angular momentum in this volume can be written in index notation as

$$
\frac{d}{d t} \int_{V_{\mathrm{M}}} \epsilon_{i j k} x_{j}\left(\rho v_{k}\right) d V=\int_{V_{\mathrm{M}}} \epsilon_{i j k} x_{j}\left(\rho g_{k}\right) d V+\int_{S_{\mathrm{M}}} \epsilon_{i j k} x_{j}\left(n_{l} \sigma_{l k}\right) d S+\int_{V_{\mathrm{M}}} c_{i} d V,
$$

where $\boldsymbol{c}(\boldsymbol{x})$ is the torque density field (also called a couple density field or a body torque field).

(a) Use the divergence theorem to show that

$$
\int_{S_{\mathrm{M}}} \epsilon_{i j k} x_{j}\left(n_{l} \sigma_{l k}\right) d S=\int_{V_{\mathrm{M}}}\left(\epsilon_{i l k} \sigma_{l k}+\epsilon_{i j k} x_{j}\left(\frac{\partial \sigma_{l k}}{\partial x_{l}}\right)\right) d V
$$

(b) Use this result along with (1.66) to show that

$$
\epsilon_{i l k} \sigma_{l k}+c_{i}=0
$$

If there are no couples, $c_{i}=0$ and the only general way to satisfy this equation is to take $\sigma_{l k}=\sigma_{k l}$. Therefore, in the absence of external torques, the stress tensor must be symmetric. Furthermore, multiplying by $\epsilon_{i m n}$ and using (A.8) yields that

$$
\sigma_{m n}-\sigma_{n m}+\epsilon_{i m n} c_{i}=0
$$

so even in the presence of external torques, the stress tensor can only be asymmetric if it depends on $\boldsymbol{c}$ in the specific manner shown here. 


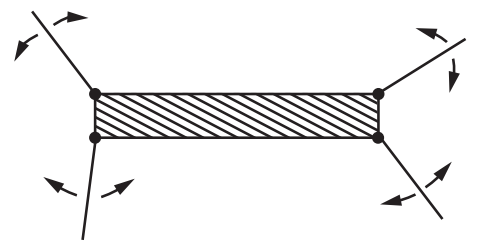

Figure 1.10 A microswimmer. Each limb can pivot around its junction with the body in the plane of the page.

1.7 A friend of yours who is a whiz at nanofabrication has constructed a small "swimming" device made of rods connected by hinges such that the "limbs" of the device can be moved by tiny motors - see Figure 1.10. Each limb can only move in the plane of the paper. But alas, your friend doesn't know any fluid mechanics - the protocol that was designed to sequentially move the limbs doesn't work, and the "swimmer" flops pathetically back and forth in the fluid. Design a limb motion protocol (i.e., a repeating sequence of limb motions) that will move the swimmer in one direction on average at zero Reynolds number. Also, give an example of a protocol that will not lead to any net motion.

1.8 (a) Show that for viscous incompressible flow the rate of conversion of mechanical to internal energy

$$
\Phi_{v}=\int_{V_{\mathrm{D}}} \sigma: \nabla v d V
$$

is nonnegative.

(b) Show that for an incompressible flow

$$
\int_{V_{\mathrm{D}}} \mathbf{E}: \nabla \boldsymbol{v} d V=\int_{V_{\mathrm{D}}} \mathbf{E}: \mathbf{E} d V,
$$

where $\mathbf{E}$ is the deformation rate. It will be easier to work with the second expression in the next part of the problem.

(c) Now consider incompressible velocity fields $\boldsymbol{v}$ and $\boldsymbol{v}^{\prime}$, where $\boldsymbol{v}$ solves the Stokes equation and $\boldsymbol{v}^{\prime}$ does not (but both $\boldsymbol{v}$ and $\boldsymbol{v}^{\prime}$ satisfy the same boundary conditions). Show that the dissipation rate for $\boldsymbol{v}^{\prime}$ is always greater than or equal to that for $\boldsymbol{v}$. Start with the identity that

$$
\int_{V_{\mathrm{D}}} \mathbf{E}^{\prime}: \mathbf{E}^{\prime} d V-\int_{V_{\mathrm{D}}} \mathbf{E}: \mathbf{E} d V=\int_{V_{\mathrm{D}}}\left(\mathbf{E}^{\prime}-\mathbf{E}\right):\left(\mathbf{E}^{\prime}-\mathbf{E}\right) d V+2 \int_{V_{\mathrm{D}}}\left(\mathbf{E}^{\prime}-\mathbf{E}\right): \mathbf{E} d V
$$

and show that the right-hand side is nonnegative. This completes the proof that the Stokes equation yields the flow that minimizes dissipation.

Hint: Notice that

$$
\frac{\partial\left(v_{i}-v_{i}^{\prime}\right)}{\partial x_{j}} E_{i j}=\frac{\partial}{\partial x_{j}}\left(\left(v_{i}-v_{i}^{\prime}\right) E_{i j}\right)-\left(v_{i}-v_{i}^{\prime}\right) \frac{\partial E_{i j}}{\partial x_{j}} .
$$

The first of these terms is a divergence and the second contains $\frac{\partial E_{i j}}{\partial x_{j}}=\frac{1}{2} \nabla^{2} v_{i}$. 